\title{
Long term mortality and risk of end-stage renal disease following acute rrt in icu patients
}

\author{
R Lohse ${ }^{{ }^{*}}$, MB Damholt ${ }^{2}$, J Wiis $^{3}$, A Perner $^{3}$, T Lange $^{4}$, M Ibsen $^{3}$ \\ From ESICM LIVES 2015 \\ Berlin, Germany. 3-7 October 2015
}

\section{Introduction}

In the intensive care unit (ICU) the need for acute renal replacement therapy (aRRT) is associated with high mortality and risk of end-stage renal disease (ESRD). We investigated long term mortality and progression to ESRD in ICU patients requiring aRRT and factors associated to these.

\section{Methods}

Retrospective analysis of all adult patients admitted to the general ICU, Rigshospitalet, from 1/1-2005 to 31/122012, identified through the ICU database, excluding chronic dialysis patients. ESRD was defined as the need of dialysis $>90$ days or kidney transplant.

\section{Results}

Of 5766 patients included, 931 (16\%) received aRRT, $4762(83 \%)$ did not receive any RRT and 73 (1\%) only received aRRT during a later ICU admission. The 90day mortality was $55 \%$ for aRRT patients and $22 \%$ for those not requiring aRRT $(\mathrm{p}<0.001)$. The median (IQR) age was $61(49-68)$ for aRRT 90-day survivors and $65(55-73)$ for aRRT non-survivors $(\mathrm{p}<0.001)$. The median SAPS II score was $53(43$ - 64) for aRRT 90-day survivors and $61(49-74)$ for aRRT non-survivors $(\mathrm{p}<0.001)$. Characteristics of aRRT patients developing ESRD and those that did not, are displayed in tables 1-3. The 7-year risk of ESRD for patients surviving 90 days after admission was $10 \%(7$ - 14) for aRRT patients as compared to $0.5 \%$ (95\% CI $0.3-0.9)$ for those not receiving aRRT $(\mathrm{p}<0.001)$.

\section{Conclusions}

The aRRT patients surviving 90 days were younger and less severely ill at ICU admission as compared to the

Table 1 Baseline characteristics

\begin{tabular}{|c|c|c|c|c|c|c|}
\hline & $\begin{array}{c}\text { Data } \\
\text { available }\end{array}$ & $\begin{array}{l}90 \text { day survivors with ESRD } \\
(\mathrm{N}=38)\end{array}$ & $\begin{array}{l}90 \text { day survivors without ESRD } \\
(\mathrm{N}=404)\end{array}$ & & $P$ & \\
\hline Age (years), median (IQR) & 442 & 61 & $(52.0-70.0)$ & 61 & $(49-68)$ & 0.400 \\
\hline Gender (male) & 442 & 25 & $(65.8 \%)$ & 271 & $(67.1 \%)$ & 0.999 \\
\hline Days in the ICU, median (IQR) & 442 & 7.2 & $(3.6-15.6)$ & 10.9 & $\begin{array}{l}(5.7- \\
20.9)\end{array}$ & 0.087 \\
\hline $\begin{array}{l}\text { SAPS \|| score (points), median } \\
\text { (IQR) }\end{array}$ & 427 & 56 & $(41-68)$ & 53 & $(43-64)$ & 0.39 \\
\hline $\begin{array}{l}\text { APACHE II score (points), median } \\
\text { (IQR) }\end{array}$ & 427 & 30 & $(25-35)$ & 26 & $(21-31)$ & 0.009 \\
\hline First SOFA (points), median (IQR) & 417 & 11 & $(8-15)$ & 12 & $(9-14)$ & 0.861 \\
\hline MAX SOFA (points), median (IQR) & 418 & 12 & $(9-15)$ & 13 & $(11-16)$ & 0.377 \\
\hline$\underline{\text { Vasopressor treatment }}$ & 442 & 24 & $(63.2 \%)$ & 297 & $(73.5 \%)$ & 0.279 \\
\hline Mechanical ventilation & 442 & 30 & $(78.9 \%)$ & 371 & $(91.8 \%)$ & 0.040 \\
\hline
\end{tabular}

${ }^{1}$ Copenhagen University Hospital - Rigshospitalet, Department of Intensive

Care 4131, Copenhagen, Denmark

Full list of author information is available at the end of the article 
Table 2. Preexisting Comorbidity

\begin{tabular}{|c|c|c|c|c|c|c|}
\hline & $\begin{array}{c}\text { Data } \\
\text { available }\end{array}$ & $\begin{array}{l}90 \text { day survivors with ESRD } \\
(\mathrm{N}=38)\end{array}$ & $\begin{array}{l}90 \text { day survivors without ESRD } \\
(\mathrm{N}=404)\end{array}$ & & $P$ & \\
\hline $\begin{array}{l}\text { Chronic kidney disease (non- } \\
\text { dialysis) }\end{array}$ & 442 & 23 & $(60.5 \%)$ & 90 & $(22.3 \%)$ & $\begin{array}{c}< \\
0.001 \\
\end{array}$ \\
\hline Hypertension & 442 & 14 & $(36.8 \%)$ & 119 & $(29.5 \%)$ & 0.505 \\
\hline Diabetes & 442 & 6 & $(15.8 \%)$ & 56 & $(13.9 \%)$ & 0.784 \\
\hline Congestive heart failure & 442 & 4 & $(10.5 \%)$ & 39 & $(9.7 \%)$ & 0.993 \\
\hline Peripheral vascular disease & 442 & 9 & $(23.7 \%)$ & 82 & $(20.3 \%)$ & 0.734 \\
\hline Cerebrovascular disease & 442 & 3 & $(7.9 \%)$ & 36 & $(8.9 \%)$ & 0.968 \\
\hline Malignant neoplasm & 442 & 12 & $(31.6 \%)$ & 83 & $(20.5 \%)$ & 0.180 \\
\hline Ischemic heart disease & 442 & 4 & $(10.5 \%)$ & 29 & $(7.2 \%)$ & 0.783 \\
\hline $\begin{array}{l}\text { Chronic obstructive pulmonary } \\
\text { disease }\end{array}$ & 442 & 4 & $(10.5 \%)$ & 50 & $(12.4 \%)$ & 0.928 \\
\hline
\end{tabular}

Table 3. Primary diagnosis during ICU stay

\begin{tabular}{|c|c|c|c|c|c|c|}
\hline & $\begin{array}{c}\text { Data } \\
\text { available }\end{array}$ & $\begin{array}{l}90 \text { day survivors with ESRD } \\
(\mathrm{N}=38)\end{array}$ & $\begin{array}{l}90 \text { day survivors without ESRD } \\
(\mathrm{N}=404)\end{array}$ & & $P$ & \\
\hline Sepsis & 442 & 7 & $(18.4 \%)$ & 112 & $(27.7 \%)$ & 0.466 \\
\hline Other infectious diseases & 442 & 6 & $(15.8 \%)$ & 20 & $(5.0 \%)$ & 0.025 \\
\hline Endocrinological diseases & 442 & & $(0.0 \%)$ & 3 & $(0.7 \%)$ & 0.868 \\
\hline Cardiovascular diseases & 442 & 3 & $(7.9 \%)$ & 43 & $(10.6 \%)$ & 0.869 \\
\hline Respiratory diseases & 442 & 9 & $(23.7 \%)$ & 66 & $(16.3 \%)$ & 0.514 \\
\hline $\begin{array}{l}\text { Gastrointestinal or liver } \\
\text { diseases }\end{array}$ & 442 & 2 & $(5.3 \%)$ & 11 & $(2.7 \%)$ & 0.675 \\
\hline Trauma or poisoning & 442 & 4 & $(10.5 \%)$ & 38 & $(9.4 \%)$ & 0.975 \\
\hline Other & 442 & 7 & $(18.4 \%)$ & 111 & $(27.4 \%)$ & 0.483 \\
\hline
\end{tabular}

non-survivors. The survivors developing ESRD had more frequently preexisting chronic kidney disease and higher APACHE II scores as compared to those who did not develop ESRD. aRRT patients had increased risk of ESRD up to 7 years after ICU admission, emphasizing the KDIGO recommendations to follow up AKI patients due to their increased risk for CKD.

\footnotetext{
Authors' details

${ }^{1}$ Copenhagen University Hospital - Rigshospitalet, Department of Intensive Care 4131, Copenhagen, Denmark. ${ }^{2}$ Copenhagen University Hospital, Rigshospitalet, Department of Nephrology 2132, Copenhagen, Denmark. ${ }^{3}$ Copenhagen University Hospital, Rigshospitalet, Department of Intensive Care 4131, Copenhagen, Denmark. ${ }^{4}$ University of Copenhagen, Section of Biostatistics, Copenhagen, Denmark.
}

Published: 1 October 2015

doi:10.1186/2197-425X-3-S1-A11

Cite this article as: Lohse et al: Long term mortality and risk of

end-stage renal disease following acute rrt in icu patients. Intensive Care

Medicine Experimental 2015 3(Suppl 1):A11.

\section{Submit your manuscript to a SpringerOpen ${ }^{\circ}$ journal and benefit from:}

- Convenient online submission

- Rigorous peer review

- Immediate publication on acceptance

- Open access: articles freely available online

- High visibility within the field

- Retaining the copyright to your article

Submit your next manuscript at $>$ springeropen.com 